\title{
Real-time Polymerase Chain Reaction System Using an Open Platform Camera
}

\author{
Seul-Bit-Na Koo, ${ }^{1,2}$ Chan-Young Park, ${ }^{1,2}$ Yu-Seop Kim, ${ }^{1,2}$ \\ Hye-Jeong Song, ${ }^{1,2}$ and Jong-Dae Kim ${ }^{1,2^{*}}$ \\ ${ }^{1}$ Department of Convergence Software, Hallym University, \\ 1 Hallymdaehak-gil, Chuncheon-si, Gangwon-do 24252, Korea \\ ${ }^{2}$ Bio-IT Research Center, Hallym University, \\ 1 Hallymdaehak-gil, Chuncheon-si, Gangwon-do 24252, Korea \\ (Received May 31, 2018; accepted October 24, 2018)
}

Keywords: real-time PCR, fluorescence detection, open platform, Raspberry Pi

The DNA amplified by the conventional polymerase chain reaction (PCR) is subjected to two-step detection, electrophoresis, and gel image analysis. Recently, real-time PCR has been developed to detect DNA amplification by measuring fluorescence in real time without the need for a conventional detection process. Real-time PCR systems require thermal cyclers for DNA amplification and fluorescence measurement systems to measure fluorescence in real time. Some of these systems measure fluorescence with a camera. However, owing to the simultaneous handling of fluorescence excitation and emission for dozens of tubes, there is a limit to the size, cost, and assembly of optics. Recently, cameras have been continuously developed as imaging devices for rapidly developing open platforms, and their performance has also been rapidly improved. In this paper, we propose a low-cost, compact fluorescence detection system for real-time PCR systems using open platforms and cameras. To simplify the optics, the entire tube was divided into four quadrants to minimize the field of view. In addition, the images of the tubes far from the field-of-view center were overlapped to compensate for the reduction in signal-to-noise ratio due to oblique photography. The proposed system measured the fluorescence detection performance on the basis of the amount of DNA using various fluorescent substances.

\section{Introduction}

Polymerase chain reaction (PCR) is a molecular biological technique that replicates and amplifies a desired portion of DNA with a very small amount of DNA solution. ${ }^{(1-7)}$ The PCR process is largely divided into three steps: extraction, amplification, and analysis. In the analysis, there is a two-step detection procedure to determine whether the desired part is correctly amplified. First, gel electrophoresis is performed to compare the sizes of PCR amplification products for analysis. Second, the DNA fragments of the gel that have undergone electrophoresis cannot be visualized, and the images taken using the gel documentation system

*Corresponding author: e-mail: kimjd@hallym.ac.kr https://doi.org/10.18494/SAM.2019.2096 
must be analyzed. In the conventional DNA analysis, the above procedure is performed sequentially using each corresponding instrument. In recent years, real-time PCR, with conventional DNA analysis processes integrated, has been developed. ${ }^{(6,8-11)}$ Real-time PCR amplifies and quantifies target DNA molecules simultaneously using fluorescent dye. Unlike the conventional DNA analysis, by which DNA can be observed through a detection method using gels in the saturation state after the last cycle, real-time PCR monitors and quantitatively analyzes the amplification process in real time in the exponentially growing region (polymerase) within one cycle. Although gels are used to detect saturated DNA after the last thermal cycle in the conventional DNA analysis, real-time PCR monitors and quantifies the amplification process in real time in regions where the DNA grows exponentially. With this device, smaller samples can be measured and precisely quantified. As the conventional method of detecting DNA becomes unnecessary, the analysis is completed in tubes, and the failure rate is lower than that of the conventional method; thus, the analysis can be performed rapidly. Since no further experiments are needed, the entire experiment is simplified and the risk of contamination is reduced.

Many real-time PCRs consist of a thermocycler for amplification and a camera optical system for analysis..$^{(9,12)}$ They use a fluorescent material that absorbs light energy with a specific wavelength and emits light with a longer wavelength. Fluorescent materials are divided into two categories, one is the intercalator, and the other is the probe type using the form of a fluorescence primer. If the latter probe type is used, fluorescence resonance energy transfer (FRET) occurs owing to the relationship between a fluorescent material and a quencher. ${ }^{(9)}$ In other words, considering the probe type, the camera optic system should be equipped with unique excitation and emission wavelengths. However, the cost of each consumable part of the above system is high. Analyzing all wells under the same conditions at once requires expensive optics and complex assemblies. If the wells are partitioned into several groups so that the field of view of each group is sufficiently small, and if it is possible to synthesize photos to obtain an image of all the wells, then the optics can be compact and inexpensive. However, there has between no research on this.

Open platforms evolve owing to the smart telecommunication technology and the radically developed information technology..$^{(13-15)}$ In recent years, many open platforms have been developed and can be easily used with open software in image analysis after PCR detection. As high-performance smartphone cameras are constantly being developed for open platforms, the field of open imaging devices also rapidly evolves. In particular, backlight CMOS sensor technology has been developed, and the manufacturing cost has been reduced; thus, a very small camera with good performance suitable for a portable device has also been developed.

In this paper, we propose a detection system that achieves low cost and compactness in accordance with the above trends. Using an ultrasmall camera with an open platform, the cost of the imaging system is decreased. In addition, the $5 \times 5$ wells were divided into four quadrants of $3 \times 3$ wells in one quadrant to reduce the field of view such that the optical ratio was considerably reduced. Although the excitation light was weakened in the middle portion of the wells, the signal-to-noise ratio was compensated through the synthesis process where a number of quadrant images were superimposed for that portion. The performance of the 
proposed system was verified using double-distilled water (DDW) and a fluorescent solution, whose fluorescence density was determined to simulate fluorescence brightness when the DNA was fully amplified. Experimental results showed that the composite image of four quadrants represented the overall tube image very well. The relative gain calculated from the composite image of DDW and fluorescence tubes was above 1.7, indicating a strong possibility of real-time fluorescence detection.

\section{Materials and Methods}

\subsection{Experimental device system}

Table 1 shows the main components used in the experiment. Raspberry Pi Zero W v1.1, the most popular single-board computer (SBC), and Raspberry Pi Camera v1.3 were used for the imaging system. As the SBC is very small but powerful, we can use the Python programming language to process images and collect the processed data very easily. Developed for the mobile phone camera market, the camera produces an 8/10-bit RGB/RAW image output. It has good portability with low cost and power. By using the wireless LAN function of the Raspberry Pi Zero W Board, we can connect the imaging system wirelessly to reduce the interference between the cables in the moving part.

The step motor shown in Table 1 was used to accurately divide the $5 \times 5$ tubes into four quadrants in the form of $3 \times 3$ tubes. The motor driver was connected to the PC via RS-485 communication to control the motor motion. Through the motion program, the position of each quadrant from the home position was found and stored.

For fluorescence measurement, a filter based on the green fluorescence emission of fluorescein was used. The fluorescent dye used in this experiment was 6-FAM phosphoramidite (FAM). Therefore, we selected excitation and emission filters as shown in the table. The optical system was made smaller and simpler by customizing the excitation and emission filters to be $2 \times 2$ and $4 \times 4 \mathrm{~mm}^{2}$ with $1 \mathrm{~mm}$ thickness, respectively. Figures 1 (a) and 1 (b) show the imaging system that includes the SBC and the camera with the filters. The lower holes in the pictures are for connecting the imaging system to the stepper motor so that the imaging system

can rotate. A camera with an emission filter is located above the hole. Figure 1(b) shows the other side of the imaging system with Raspberry Pi Zero W on it. For fluorescence excitation, the blue LED shown in Table 1 was used. Two LEDs with a viewing angle of $30^{\circ}$ and a

Table 1

System components.

\begin{tabular}{|c|c|c|}
\hline Imaging system & \multicolumn{2}{|c|}{ Raspberry Pi Zero W Board, Raspberry Pi Camera v1.3 } \\
\hline Motor and driver & \multicolumn{2}{|c|}{ Ezi-STEP Plus-R MINI 28 Series (Fastech Co., Ltd.) } \\
\hline Fluorescent reagent & \multicolumn{2}{|c|}{ Probe-type FAM $(0.35$ pMole $/ \mu 1)$} \\
\hline Control reagent & \multicolumn{2}{|c|}{ DDW } \\
\hline Filter & Excitation & CW: $470 \mathrm{~nm}$, BW: $40 \mathrm{~nm}$ (Chroma Technology Corp.) \\
\hline & Emission & CW: 535 nm, BW: $50 \mathrm{~nm}$ (Chroma Technology Corp.) \\
\hline Light & Blue LED & $\mathrm{CW}: 470 \mathrm{~nm}, 12000 \mathrm{mcd}, 30^{\circ}$ view angle \\
\hline
\end{tabular}




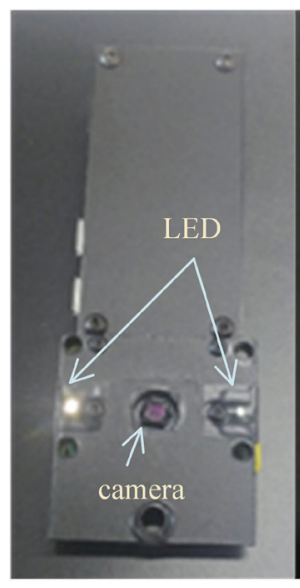

(a)

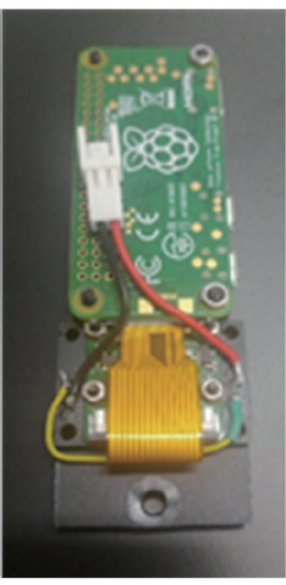

(b)

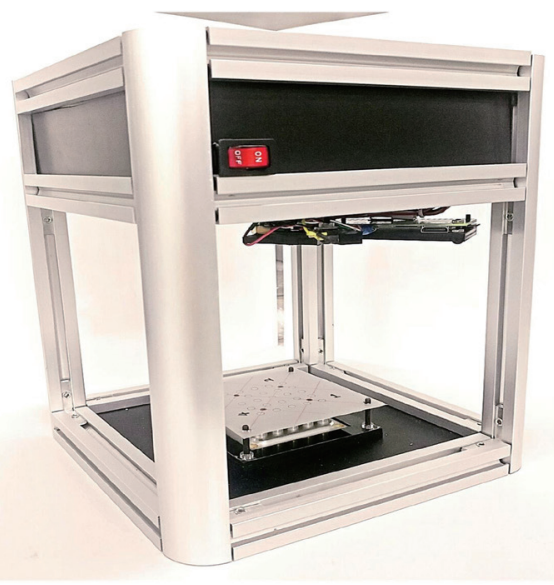

(c)

Fig. 1. (Color online) Imaging and entire experimental systems. (a) Front of the imaging system, (b) back side of the imaging system, and (c) entire experimental system.

diameter of $3 \mathrm{~mm}$ were fixed in the same direction on both sides of the camera as shown in Fig. 1(a). The LEDs uniformly illuminate $3 \times 3$ tubes of each quadrant. In this manner, the system is not only compact but also easy to assemble because there is no need to adjust the angle of the LED lighting direction. To prevent the photobleaching of fluorescence, the LED was connected to the output port of the motor driver so that it could only be turned on when shooting.

Figure 1(c) shows the entire experimental system. The distance between the tube and the camera, that is, the shooting height of each quadrant, should be kept constant. Also, the entire system should not be shaken when the motor moves and should not be tilted by the weight of the imaging system. Therefore, the frame was made of aluminum, which is robust and easy to cut. Considering a shooting distance of $135 \mathrm{~mm}$, the height of the aluminum frame was cut to $240 \mathrm{~mm}$, so that all the components could be stacked together. In the lower part of the system shown in the picture, an aluminum block for PCR thermal cycling and a mark sheet for the precise determination of the position of each quadrant were placed on the aluminum block. In the middle of the system, the imaging system hung down and the top of the screen was covered with black acrylic containing a stepper motor, a driver, and a power supply. The optics was completely made of black matt acrylic to prevent light reflection.

\subsection{Experimental method}

In this paper, we shot 25 wells of $5 \times 5$ divided into four $3 \times 3$ quadrants to minimize the optics. Since each quadrant contains $3 \times 3$ wells, the middle well is duplicated in all four quadrant images, and the middle row and column are duplicated in two adjoining quadrant images.

Figure 2 shows the illumination and imaging axes when the imaging system is positioned to capture the 1st quadrant. The orange circle is the imaging axis and green circles are illumination axes. Translucent blue circles indicate half angles of LEDs. Each yellow dotted box shows the boundary of the four wells of the corner that are most prominent in each quadrant image. 


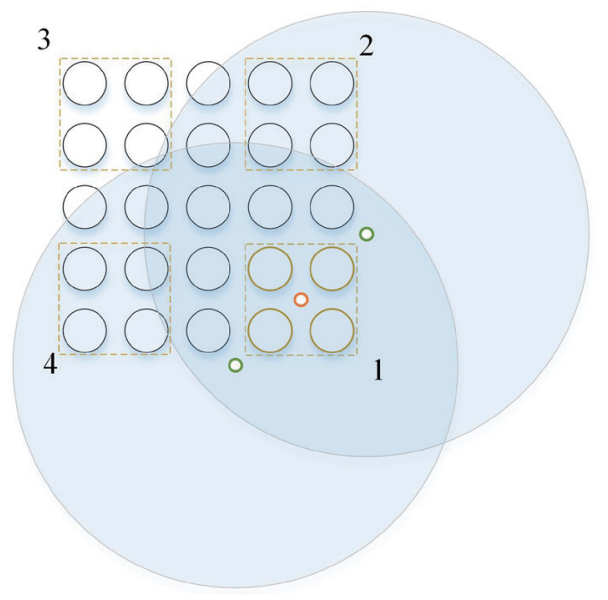

Fig. 2. (Color online) Four $5 \times 5$ quadrants of wells.

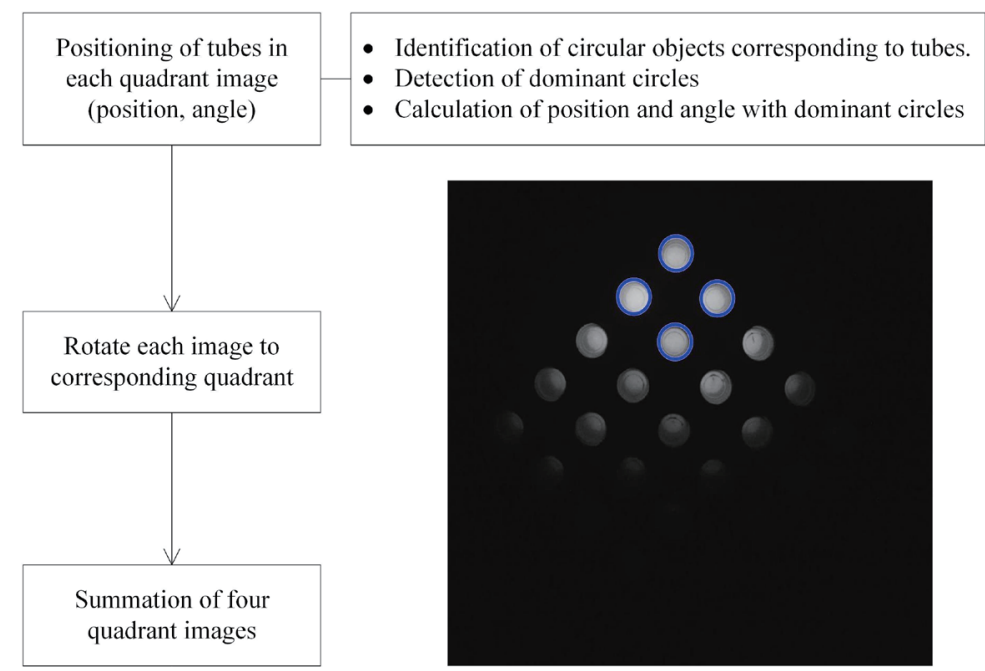

Fig. 3. (Color online) Synthesis procedure for four quadrant images and example of separation of four dominant circles at the corner.

To locate each quadrant accurately, a mark sheet with the imaging axis of each quadrant was made and placed on an aluminum block well. The position of each quadrant was detected by moving the motor so that the imaging axis of each quadrant was at the center of the image. To determine whether the stored positions were correct, the position error statistics were obtained by analyzing the images obtained by 10 visits of each position.

To emulate the PCR plateau, tubes containing $0.35 \mathrm{pmol} / \mu 1$ FAM solution were used. This solution was used to fill 25 tubes and inserted in all of the 25 wells, and four quadrant images were obtained and synthesized to obtain a FAM image. Likewise, a DDW image was obtained by taking four quadrant images by inserting tubes filled with DDW into all of the 25 wells. The four quadrant images were synthesized by the image processing shown in Fig. 3. In each quadrant image, the topmost $2 \times 2$ tubes are the brightest and are marked by blue circles in the 
image in Fig. 3. Dominant circles were observed in the image and the four major tubes at the corner were determined using their relative position information. The center and angle of the image rotation were calculated from the center of the four tubes.

\section{Results}

Figure 4 shows the images obtained by stacking quadrature images. The image on the left-hand side of the figure was taken with $0.35 \mathrm{pmol} / \mu \mathrm{FAM}$ solution in all 25 tubes. The $0.35 \mathrm{pmol} / \mu \mathrm{FAM}$ solution emulated the brightness of fluorescence when the DNA was fully amplified. The right-hand side of the figure shows a collection of images taken with DDW. As shown in the figure, the brightness of the DDW image was increased sixfold for visibility. In all quadrant images, the $2 \times 2$ tubes at the top corner are relatively bright. The positions of the corresponding tubes seem to be the same in all quadrant images. However, for the accurate synthesis of four quadrant images, the tube areas were segmented, as described in the previous section, to calculate position and diameter.

Table 2 shows the position and diameter variations of the four tubes located at the corner when the four quadrant positions were visited ten times. As shown in the first and second columns of the table, the deviation of the position between the visits was only up to 4 pixels and that of the diameter was only 1 pixel. The variation of the tube diameter or that of the distance between the tubes was also less than 2 pixels as shown in the last two columns of the table. On

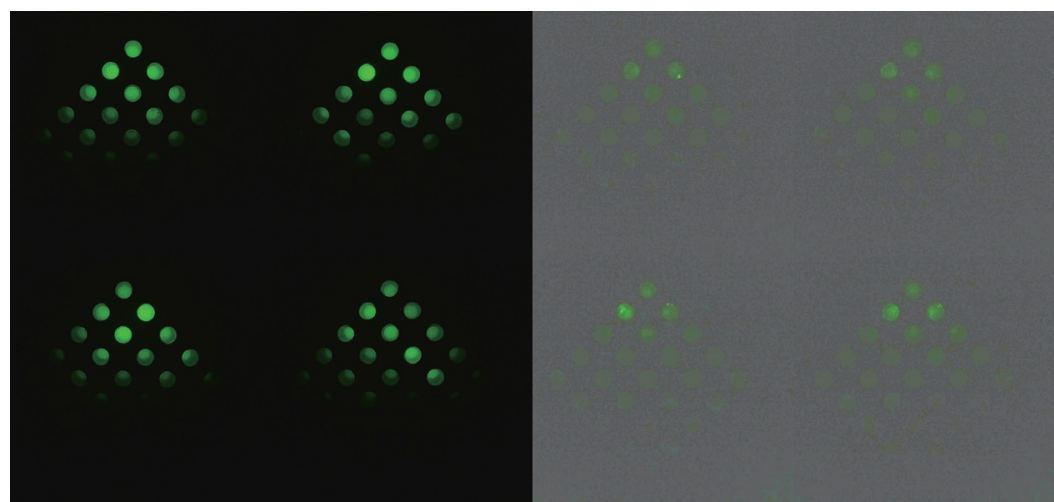

(a)

(b)

Fig. 4. (Color online) (a) Four piled quadrant images of FAM solution and (b) those of DDW. The DDW image was visually enhanced 6 times.

Table 2

Variations of tube position and diameter when four quadrant positions were visited 10 times (pixels).

\begin{tabular}{lccccc}
\hline & Position error & Diameter error & $\begin{array}{c}\text { Quadrant position } \\
\text { variation }\end{array}$ & Tube diameter & Tube distance \\
\hline Mean & 2.1 & 0.6 & 26.8 & 60.1 & 223.6 \\
Min & 0.4 & 0.2 & 15.8 & 58.8 & 222.6 \\
Max & 4.1 & 1.0 & 62.2 & 61.3 & 225.3 \\
Std. & & & & 0.2 & 1.3 \\
\hline
\end{tabular}


the other hand, the positions of the tubes between the quadrants were significantly different. The difference was up to 62 pixels, which was larger than the tube diameter as shown in the fourth column of the table. Therefore, we could not use the same rotation parameter for all the quadrant images. Instead, the rotation center and angle of each quadrant image were calculated. This was reasonable because the distance between the tubes and the tube diameter were almost constant. The yellow vector in Fig. 5 was calculated from the centers of the topmost and bottommost tubes among the four corner tubes. The center of rotation of each quadrant image was the position shifted two times by the yellow vector, which was denoted by a green arrow, from the top tube center, and the rotation angle was the difference between the yellow vector and one of the white vectors indicating the angle at which each quadrant was located. The first and second quadrant vectors shown in Fig. 5 were -45 and +45 degrees from the vertical direction of the image, respectively. The third and fourth quadrant vectors are not shown in the figure, but they were slanted at +135 and -135 degrees, respectively.

Each quadrant image was rotated so that the obtained center vector (yellow vector) matched the quadrant vector (white vector) corresponding to the quadrant. The rotated quadrant images were added to obtain a full image as shown in Fig. 6. The image on the left-hand side of the

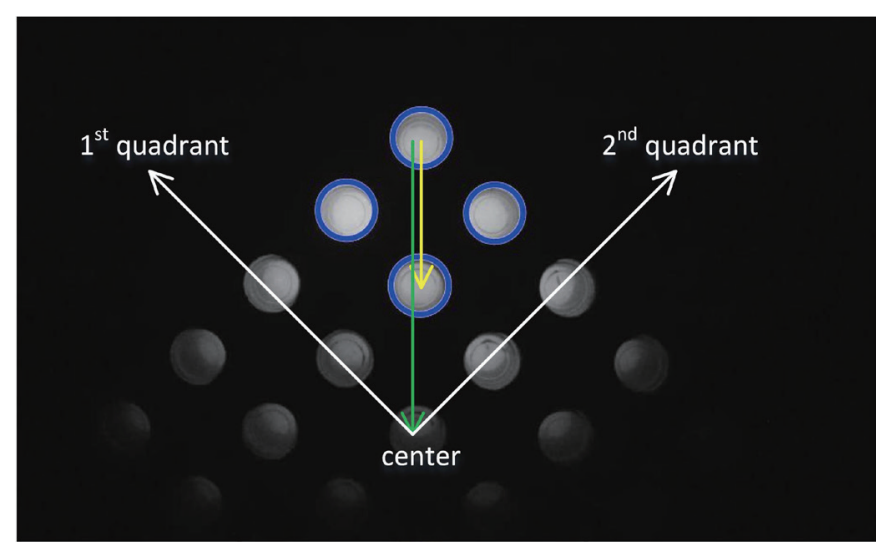

Fig. 5. (Color online) Method of determining the center and angle for rotating each quadrant image to corresponding location.

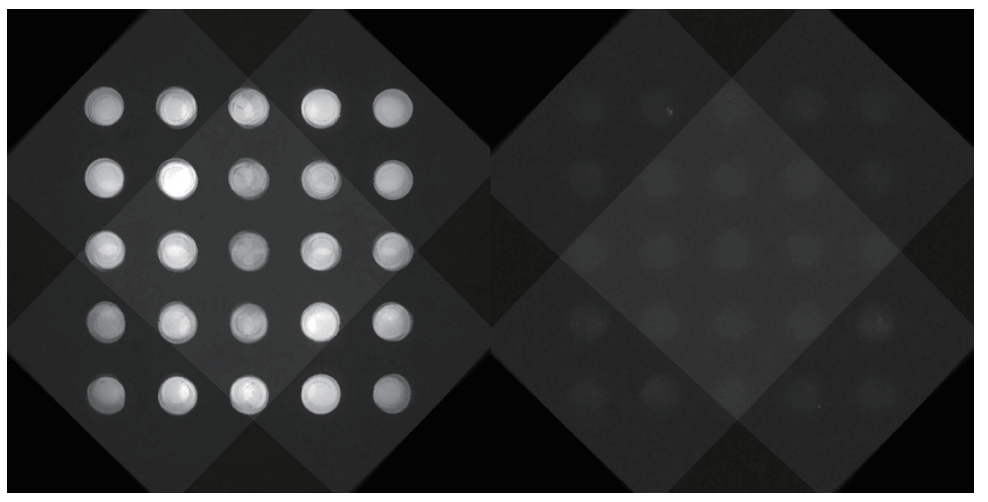

Fig. 6. Images synthesized from the quadrant images. The left image represents the FAM solution images and the right one represents the DDW images. The DDW images were enhanced 6 times. 
figure represents the FAM solution images and that on the right-hand side one represents the DDW images. The DDW images were enhanced again 6 times for visibility. Since the distance between the tubes and the tube diameter were almost constant, the positions of the tubes were calculated easily. The calculated positions are denoted by blue circles in Fig. 7. As you can see from the image, we found a good match.

Figure 8 shows the average brightness of each tube. The left graph is for the FAM solution image and the right one is for the DDW image. In the DDW image, the tube had an average brightness of about 60 and an average brightness of 200 or more in the FAM image. To calculate the relative gain at the arbitrary well position, the difference between the brightness when a FAM tube is placed at the well position and that with a DDW tube is obtained, and it is divided by the latter. It is generally more than 1.7, as shown in Fig. 9. The average brightness of the tubes with FAM and DDW and the relative gains are summarized in Table 3.

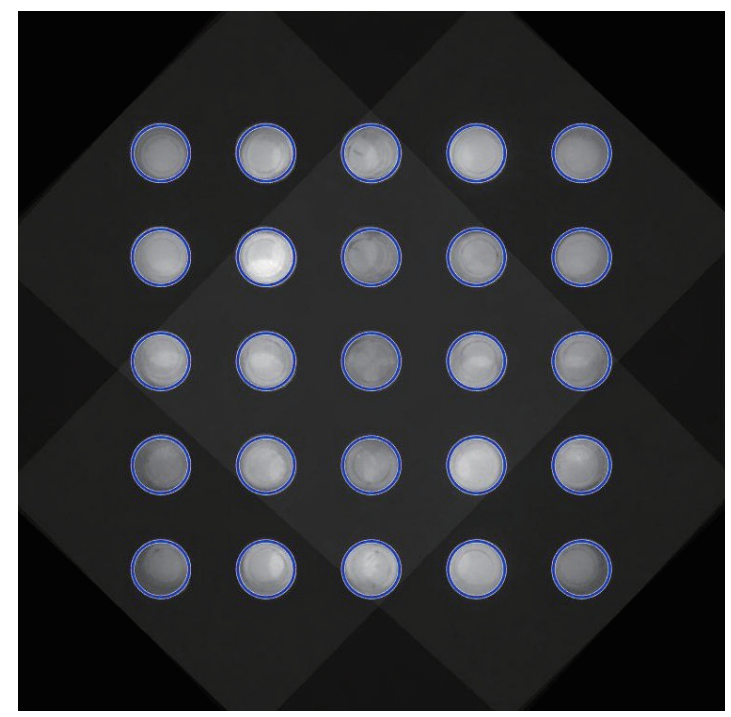

Fig. 7. (Color online) Estimated location of the tube overlaid with a blue circle on the left composite image in Fig. 3.

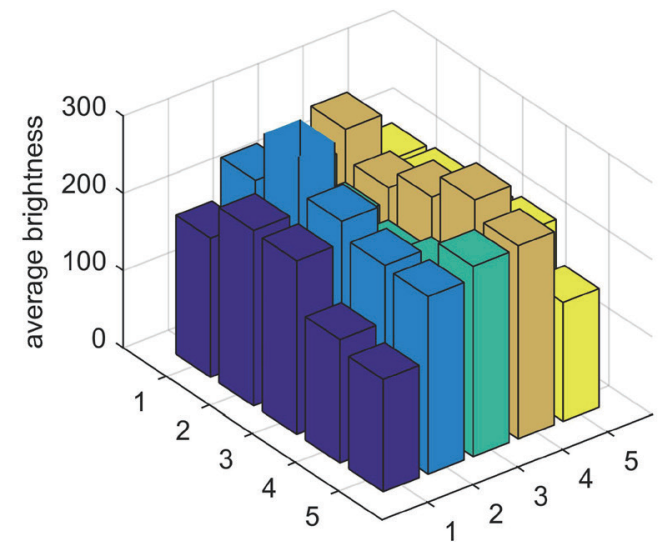

(a)

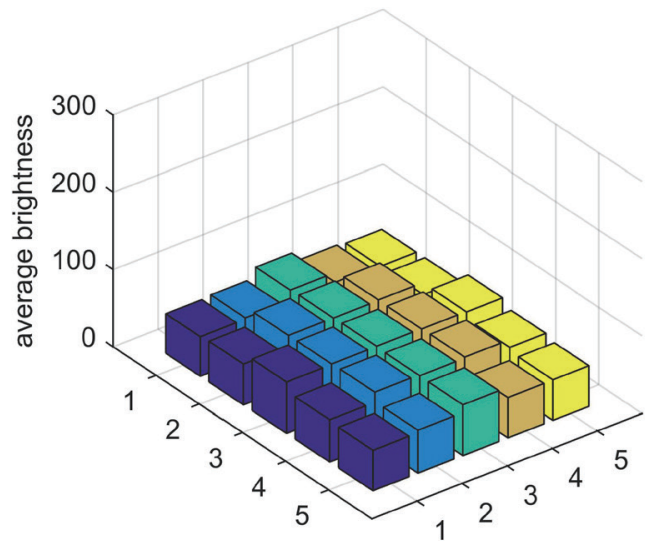

(b)

Fig. 8. (Color online) Average brightness of the tubes with (a) FAM solution and (b) DDW. 


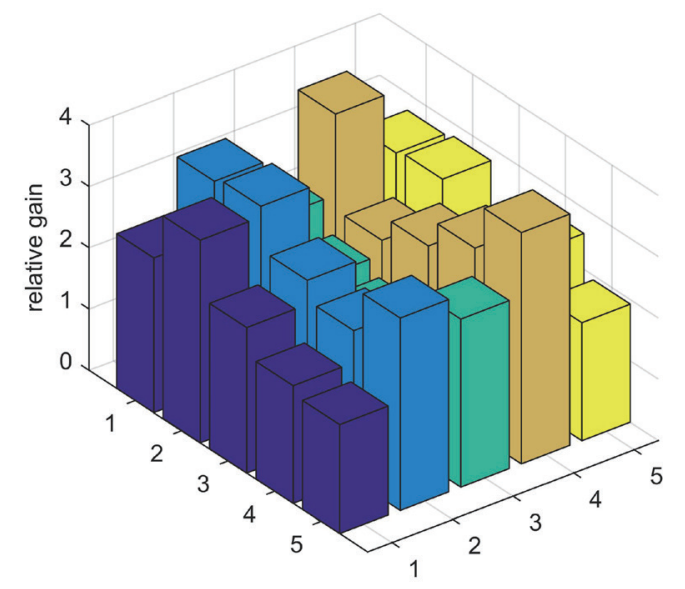

Table 3

Brightness and relative gain statistics of each tube.

\begin{tabular}{lccc}
\hline & FAM & DDW & Rel. gain \\
\hline Mean & 216.3 & 60.3 & 2.6 \\
Min & 145.3 & 51.1 & 1.7 \\
Max & 300.5 & 67.8 & 3.8 \\
\hline
\end{tabular}

Fig. 9. (Color online) Relative gains.

Given that the relative difference in relative gain is compensated by calibration, experimental results show that the fluorescence detection of real-time PCR machines with $5 \times 5$ wells is possible using inexpensive optics and a camera.

\section{Conclusions}

In this paper, we propose a low-cost implementation of a fluorescence-measuring device for real-time PCR using an open platform and a camera developed for various applications. To secure a wide field of view, a method of retaining a wide field of view by moving an imaging system with a small field of view instead of expensive optical components is proposed. To simplify motion control, we propose a method of capturing the whole well by dividing the view into four quadrants by merely rotating the motion and synthesizing them. For the region with weak fluorescence, sufficient relative gain could be obtained by the superposition of quadrant images. The fluorescence relative gain in each well was obtained using a fluorescence solution with DDW and fully amplified DNA brightness. As a result, it was concluded that the proposed method was sufficient for the fluorescence detection of real-time PCR because the relative gain was 1.7 or more in all the wells.

The proposed method can be applied to biomedical instruments for various fluorescence measurements requiring a wide field of view. They usually use a precise 2D scanner or precision optics. It is expected that the proposed method can reduce the cost and size of their optics.

\section{Acknowledgments}

This research was supported by the Basic Science Research Program through the National Research Foundation of Korea (NRF) funded by the Ministry of Science, ICT \& Future Planning (NRF-2017R1A2B4002679), and by the Leading Human Resource Training Program of Regional Neo Industry through the National Research Foundation of Korea (NRF) funded by the Ministry of Science, ICT \& Future Planning (NRF-2016H1D5A1909654). 


\section{References}

1 D. Verdoy, Z. Barrenetxea, J. Berganzo, M. Agirregabiria, J. M. Ruano-López, J. M. Marimón, and G. Olabarría: Biosens. Bioelectron. 32 (2012) 259.

2 J. Wu, R. Kodzius, K. Xiao, J. Qin, and W. Wen: Biomed. Microdevices 14 (2012) 179.

3 B. Srinivasan and S. Tung: J. Lab. Autom. 20 (2015) 365.

4 L. R. Volpatti and A. K. Yetisen: Trends Biotechnol. 32 (2014) 347.

5 X. Jiang, W. Jing, L. Zheng, S. Liu, W. Wu, and G. Sui: Lab Chip 14 (2014) 671.

6 C. Koo, M. Malapi-Wight, H. S. Kim, O. S. Cifci, V. L. Vaughn-Diaz, B. Ma, S. Kim, H. Abdel-Raziq, K. Ong, and Y.-K. Jo: PLOS ONE 8 (2013) e82704.

7 C. Zhang and D. Xing: Nucleic Acids Res. 35 (2007) 4223.

8 M. T. Dorak, Real-Time PCR (Taylor \& Francis, New York, 2007).

9 M. Arya, I. S. Shergill, M. Williamson, L. Gommersall, N. Arya, and H. R. Patel: Expert Rev. Mol. Diagn. 5 (2005) 209.

10 U. Gibson, C. A. Heid, and P. M. Williams: Genome Res. 6 (1996) 995.

11 D. Klein: Trends Mol. Med. 8 (2002) 257.

12 D. Fraga, T. Meulia, and S. Fenster: Curr. Protoc. Essent. Lab. Tech. 8 (2014) 10.3.1.

13 M. Maksimović, V. Vujović, N. Davidović, V. Milošević, and B. Perišić: Des. Issues 3 (2014) 8.

14 G. Senthilkumar, K. Gopalakrishnan, and V. S. Kumar: Int. J. Emerging Trends Technol. Comput. Sci. 3 (2014) 213.

15 T. C. Wilkes, A. J. McGonigle, T. D. Pering, A. J. Taggart, B. S. White, R. G. Bryant, and J. R. Willmott: Sensors 16 (2016) 1649. 